\title{
Deformation of a Uniform Half-Space with Rigid Boundary Caused By a Long Inclined Strike Slip Fault of Finite Width
}

\author{
Ravinder Kumar Sahrawat ${ }^{1}$, Yogita Godara ${ }^{1}$, and Mahabir Singh ${ }^{2}$ \\ ${ }^{1}$ Deenbandhu Chhotu Ram University of Science and Technology, Murthal, Sonepat-131039 \\ ${ }^{2}$ CoE, Deenbandhu Chhotu Ram University of Science and Technology, Murthal, Sonepat-131039 \\ Haryana, India
}

\begin{abstract}
Deformation of a uniform half-space with rigid boundary caused by a long inclined strike-slip fault of finite width is studied analytically and numerically. Closed-form expressions for the displacement and stresses at any point in a homogeneous, isotropic, perfectly elastic half-space with rigid boundary caused by a long inclined strike-slip fault of finite width are derived. The variation of the displacement and stresses at the interface with the distance from the fault and depth from the fault is studied numerically. It is found that the displacement and stress field is heavily dependent on the dip-angle.

Keywords: Inclined strike-slip fault; Half-space; finite width.
\end{abstract}

\section{Introduction}

Several works deal with the mathematical treatment of static elastic residual fields on the basis of dislocation theory which have been contributed to knowledge about the deformation of the Earth's crust associated with an earthquake ( Steketee (1958a, b), Chinnery (1961), Maruyama (1966)). Two dimensional dislocation models of faulting are useful to explain the observed co-seismic deformations. Strike-slip faults with very little vertical component of motion, i.e.: the slip vector is nearly parallel to the strike direction. Strike-slip faults are typically steep or vertical and in Andersonian fault theory are associated with a stress regime where both maximum and minimum stresses are near horizontal. Many strike-slip faults are idealised as being vertical cuts going, presumably, all the way to the core-mantle boundary. In truth, the geometry of apparently vertical strike-slip faults is quite variable with depth.

Wang and $\mathrm{Wu}$ (1983), following Steketee's method of integration, obtained a closed-form analytical solution for the displacement and stress field due to a "trapezodial" type of non-uniform slip along a strike-slip fault in an elastic half-space. Singh (1985) considered the problem of the static deformation of a multilayered half-space by a long strike-slip dislocation. The representation of sources corresponding to anti-plane strain has been considered. Singh and Garg (1986) obtained the integral expressions for the Airy stress function in an unbounded medium due to various two-dimensional seismic sources. Sharma and Garg (1991) have obtained the deformation field at any point of a layered half-space due to very long strike-slip dislocations. Madan and Garg (1997) obtained the analytic expressions for the displacement and stresses at any point of an orthotropic horizontal elastic layer coupling in different ways to a base due to a very long inclined strike-slip fault situated in a layer.

Kumar et al. (2002) studied static deformation of two monoclinic elastic half-spaces in welded contact due to a long inclined strike-slip fault situated in one of the half-spaces analytically and numerically. Chug et al. (2010) obtained closed-form analytical expressions for the deformation at any point of a two-phase medium consisting of a homogeneous orthotropic half-space in welded contact with a homogeneous isotropic elastic half-space caused by non-uniform slip along a very long vertical strike-slip dislocation, situated in the orthotropic elastic half-space.

Beginning with the expression for the displacement for strike-slip line source Malik and Singh (2013), we have obtained the displacement field for a long inclined strike-slip fault of finite width located at an arbitrary point of a homogeneous, isotropic, perfectly elastic half-space of rigid boundary by integration over the width of the fault. The expressions for stresses follow immediately. The closed-form expressions are used to study the variation of the displacement and stresses at the interface with the distance from the fault and depth from the fault. The inclination of the fault introduces asymmetry of various degrees in the displacement field depending on the dip-angle.

\section{Theory}

Let the Cartesian co-ordinates be denoted by $\left(x_{1}, x_{2}, x_{3}\right)$ with $x_{3}$-axis vertically upward. Consider a homogeneous, perfectly elastic half-space with rigid boundary. The half-space is assumed to be isotropic with stress-strain relation 
$p_{i j}=2 \mu e_{i j}+\lambda \delta_{i j} e_{k k}$

where, $\delta_{i j}$ is the Kronecker delta $\left(\delta_{i j}=1\right.$ for $i=j$ and $\delta_{i j}$ for $i \neq j$ ), $\lambda$ and $\mu$ are the lame constants and $e_{i j}$ is the strain tensor. If $\left(u_{1}, u_{2}, u_{3}\right)$ denote the components of the displacement vector, the strain-displacement relations are

$e_{i j}=\frac{1}{2}\left(u_{i, j}+u_{j, i}\right)$

From equations (1) and (2), we obtain the stress-displacement relations

$p_{i j}=\mu\left(u_{i, j}+u_{j, i}\right)+\lambda \delta_{i j} u_{k, k}$

Consider an anti-strain problem for which displacement components are of the form

$u_{1}=u_{1}\left(x_{2}, x_{3}\right), \quad u_{2}=0, \quad u_{3}=0$

For zero body forces, the equilibrium equation reduces to

$\frac{\partial^{2} u_{1}}{\partial x_{2}^{2}}+\frac{\partial^{2} u_{1}}{\partial x_{3}^{2}}=0$

The non-zero strain and stress components are

$e_{12}=\frac{1}{2} \frac{\partial u_{1}}{\partial y}, \quad e_{13}=\frac{1}{2} \frac{\partial u_{1}}{\partial z}$

$p_{12}=\mu \frac{\partial u_{1}}{\partial y}, \quad p_{13}=\mu \frac{\partial u_{1}}{\partial z}$

The displacement field due to a long inclined strike-slip line dislocation can be expressed in terms of the displacements due to a vertical strike-slip dislocation and a horizontal strike-slip dislocation given by Singh and Garg (1985) in the form

$u_{1}=\cos \delta u_{1}^{(13)}-\sin \delta u_{1}^{(12)}$,

where, $\delta$ is dip-angle (Fig. 1), $u_{1}^{(13)}$ is the displacement for a horizontal strike slip dislocation and $u_{1}^{(12)}$ is the displacement for a vertical strike slip dislocation.

Using the results of Malik et al.(2013), and equation (8), the expression for the displacement in a uniform halfspace with rigid boundary due to a long inclined strike-slip line dislocation parallel to the $x_{1}$ - axis and passing through the point $(0, h)$ is found to be

$u_{1}=\frac{b d s}{2 \pi}\left[\frac{\left(x_{3}-h\right) \cos \delta-x_{2} \sin \delta}{R^{2}}+\frac{\left(x_{3}+h\right) \cos \delta+x_{2} \sin \delta}{S^{2}}\right]$

Equation (9), give the displacement for a long strike-slip fault at the point $(0, h)$. However, If the line source is located at an arbitrary point $\left(y_{2}, y_{3}\right), x_{2}$ and $h$ in equation (9) should be replaced by $\left(x_{2}-y_{2}\right)$ and $y_{3}$. We will obtain,

$u_{1}=\frac{b d s}{2 \pi}\left[\frac{\left(x_{3}-y_{3}\right) \cos \delta-\left(x_{2}-y_{2}\right) \sin \delta}{R^{2}}+\frac{\left(x_{3}+y_{3}\right) \cos \delta+\left(x_{2}-y_{2}\right) \sin \delta}{S^{2}}\right]$

Where,

$b=$ displacement discontinuity,

$d s=$ width of line source,

$\delta=$ dip-angle,

$\left(x_{2}, x_{3}\right)=$ receiver location,

$R^{2}=\left(x_{2}-y_{2}\right)^{2}+\left(x_{3}-y_{3}\right)^{2}$,

$S^{2}=\left(x_{2}-y_{2}\right)^{2}+\left(x_{3}+y_{\mathbf{x}_{3}}\right)^{2}$.

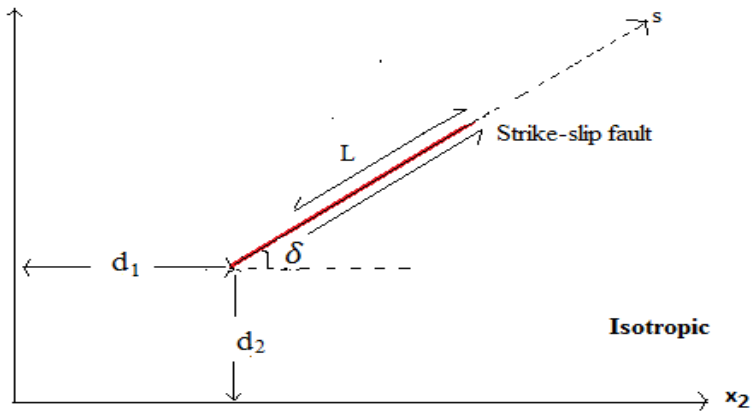

Figure 1(a). Geometry of dip-slip fault of width $L$ at a distance $d_{1}$ from $x_{3}$-axis and fro boundary of uniform half-space. 
Using polar co-ordinates $(s, \delta)$, fig.1(a)

$y_{2}=d_{1}+s \cos \delta, \quad y_{3}=d_{2}+s \sin \delta$

where, $s$ is distance from lower edge of fault measured in dip direction.

Inserting the values of $y_{2}$ and $y_{3}$ from equation (12) into equation (10) and integrating over $s$ between the limits $(0, L)$, we will obtain the following expression for the displacement for a long strike-slip fault of finite width $L$.

$u_{1}=\left.\frac{b}{2 \pi}\left[\tan ^{-1}\left(\frac{s-X \cos \delta-Y \sin \delta}{Y \cos \delta-X \cos \delta}\right)+\tan ^{-1}\left(\frac{s-X \cos \delta+Y^{\prime} \sin \delta}{Y^{\prime} \cos \delta+X \cos \delta}\right)\right]\right|_{0} ^{L}$

From equations (7) and (13), we will get the following expressions for the stresses

$p_{12}=-\left.\frac{\mu b}{2 \pi}\left[\frac{(Y-s \sin \delta)}{R^{2}}+\frac{\left(Y^{\prime}+s \sin \delta\right)}{S^{2}}\right]\right|_{0} ^{L}$
$p_{13}=\left.\frac{\mu b}{2 \pi}\left[\frac{(X-s \cos \delta)}{R^{2}}+\frac{(X-s \cos \delta)}{S^{2}}\right]\right|_{0} ^{L}$

where now,

$R^{2}=(X-s \cos \delta)^{2}+(Y-s \sin \delta)^{2}$,

$S^{2}=(X-s \cos \delta)^{2}+\left(Y^{\prime}+s \sin \delta\right)^{2}$,

$X=x_{2}-d_{1}$,

$Y=x_{3}-d_{2}$

$Y^{\prime}=x_{3}+d_{2}$

$\left.f(s)\right|_{0} ^{L}=f(L)-f(0)$.

Equations (13) - (15) give the residual elastic-field at any point of a uniform half-space with rigid boundary caused by a long inclined strike slip fault of finite width. The results for a horizontal strike-slip fault and for a vertical strike-slip fault can be obtained as a particular cases on putting $\delta=0^{\circ}$ and $\delta=90^{\circ}$, respectively.

\section{Numerical Results}

Now, we will study the behaviour of the parallel displacement $u_{1}$ and shear stresses $p_{12}, p_{13}$ at the interface $\left(x_{3}=0\right)$ for a long strike-slip fault of $\operatorname{dip} \delta$ and width $L$ and also at different distances of the fault from interface and $x_{3}$-axis.

Figure 2a-c display the variation of displacement $\left(u_{1} / b\right)$ with distance from the fault $\left(x_{2} / L\right)$ for different values of dip angle $\delta=30^{\circ}, 45^{\circ}, 60^{\circ}, 90^{\circ}$ for $x_{3}=L$ due to a long strike-slip fault located at a distance (a) at origin of interface (b) $d_{2}=L$ from the interface and at distance $d_{1}=L$ from $x_{3}$-axis (c) $d_{2}=L$ from the interface on $x_{3}$ - axis. In all figures, displacement is asymmetric about $x_{2}=0$ and also discontinuous at $x_{2}=0$ in fig. $2 \mathrm{a}, 2 \mathrm{c}$ and at $x_{2}=L$ in fig. $2 \mathrm{~b}$ depending on the position of the fault. It is noted that the magnitude of displacement decreases as dip-angle increases.

Figure 3a-c display the variation of displacement $\left(u_{1} / b\right)$ with depth from the fault $\left(x_{3} / L\right)$ for different values of dip angle $\delta=30^{\circ}, 45^{\circ}, 60^{\circ}, 90^{\circ}$ for $x_{2}=L$ due to a long strike-slip fault located at a distance (a) at origin of interface (b) $d_{2}=L$ from the interface and at distance $d_{1}=L$ from $x_{3}$ - axis (c) $d_{2}=L$ from the interface on $x_{3}$ - axis. In all figures, displacement is asymmetric about $x_{3}=0$ and discontinuous at $x_{3}=-L$ and at $x_{3}=L$ in fig. $3 \mathrm{~b}$, otherwise continuous depending on the position of the fault. It is noted that the magnitude of displacement decreases as dip-angle increases.

Figure 4a-c display the variation of shear stress $\left(L p_{12} / \mu b\right)$ with distance from the fault $\left(x_{2} / L\right)$ for different values of dip angle $\delta=30^{\circ}, 45^{\circ}, 60^{\circ}, 90^{\circ}$ for $x_{3}=L$ due to a long strike-slip fault located at a distance (a) at origin of interface (b) $d_{2}=L$ from the interface and at distance $d_{1}=L$ from $x_{3}$-axis (c) $d_{2}=L$ from the interface on $x_{3}$-axis. In all figures, displacement is asymmetric about $x_{2}=0$. It is noted that the magnitude of displacement decreases as dip-angle increases. In fig $4 \mathrm{~b}$ an $\mathrm{d} 4 \mathrm{c}$ have the same pattern. Also stress tends to zero as $x_{2}$ approaches to infinity.

Figure 5a-b display the variation of shear stress $\left(L p_{12} / \mu b\right)$ with depth from the fault $\left(x_{3} / L\right)$ for different values of dip angle $\delta=30^{\circ}, 45^{\circ}, 60^{\circ}, 90^{\circ}$ for $x_{2}=L$ due to a long strike-slip fault located at a distance (a) at origin of interface (b) $d_{2}=L$ from the interface on $x_{3}$ - axis. In all figures, displacement is asymmetric about $x_{3}=0$. It is noted that the magnitude of displacement decreases as dip-angle increases. Also stress tends to zero as $x_{3}$ approaches to infinity.

Figure 6a-b display the variation of shear stress $\left(L p_{13} / \mu b\right)$ with distance from the fault $\left(x_{2} / L\right)$ for different values of dip angle $\delta=30^{\circ}, 45^{\circ}, 60^{\circ}, 90^{\circ}$ at interface breakinf fault due to a long strike-slip fault located at a 
distance (a) $d_{2}=L$ from the interface on $x_{3}$ - axis (b) $d_{2}=L$ from the interface and at distance $d_{1}=L$ from $x_{3}$ - axis. In all figures, displacement is asymmetric about $x_{2}=0$. It is noted that the magnitude of displacement decreases as dip-angle increases. Both fig. have the same pattern. Also stress tends to zero as $x_{2}$ approaches to infinity.

Figure 7a-c display the variation of shear stress $\left(L p_{13} / \mu b\right)$ with depth from the fault $\left(x_{3} / L\right)$ for different values of dip angle $\delta=30^{\circ}, 45^{\circ}, 60^{\circ}, 90^{\circ}$ for $x_{2}=L$ due to a long strike-slip fault located at a distance (a) at origin of interface (b) $d_{2}=L$ from the interface and at distance $d_{1}=L$ from $x_{3}$ - axis (c) $d_{2}=L$ from the interface on $x_{3}$ - axis .In all figures, displacement is symmetric about $x_{3}=0$. It is noted that the magnitude of displacement decreases as dip-angle increases. Both fig. have the same pattern. Also stress tends to zero as $x_{3}$ approaches to infinity.
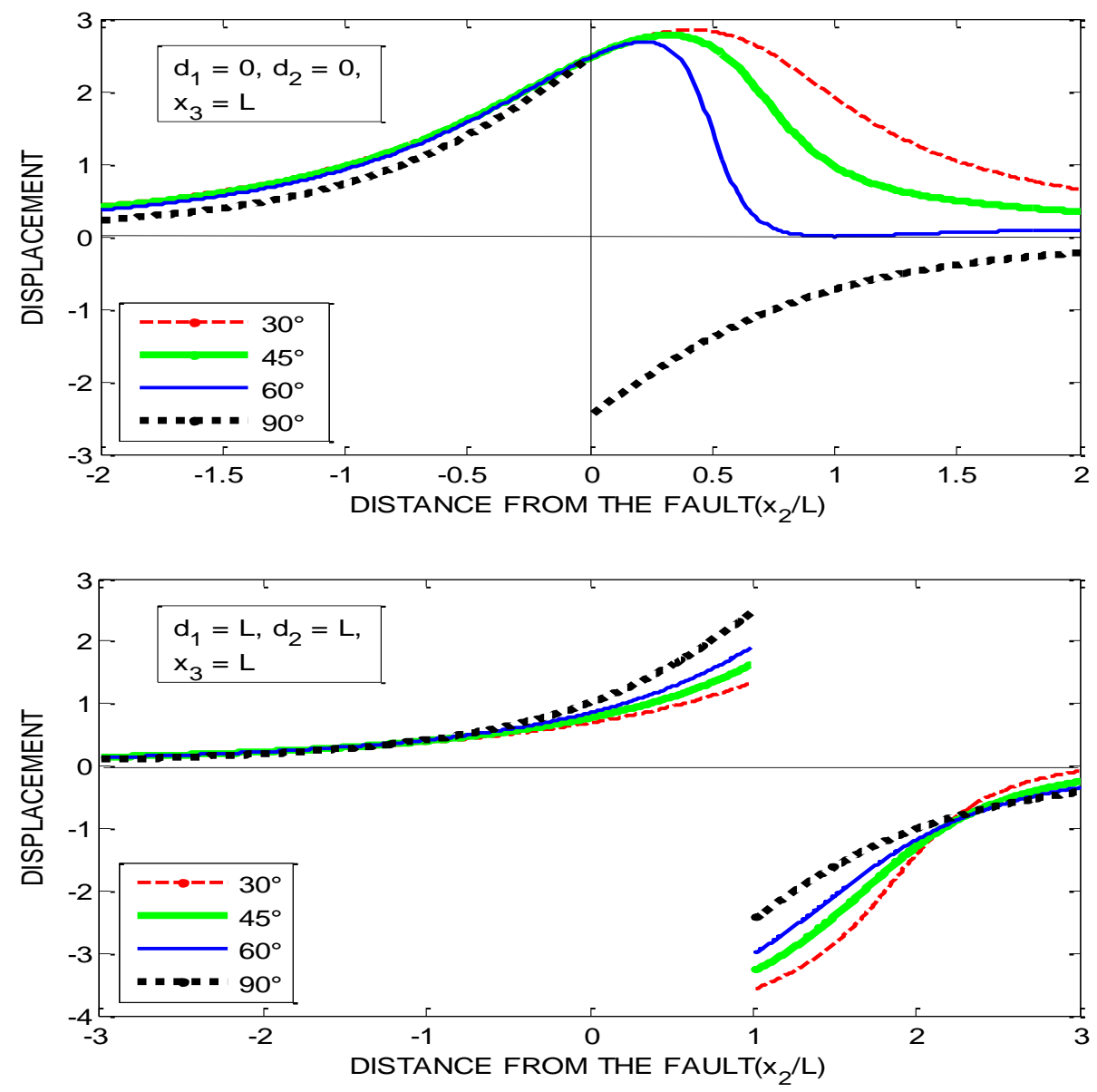

2(b)

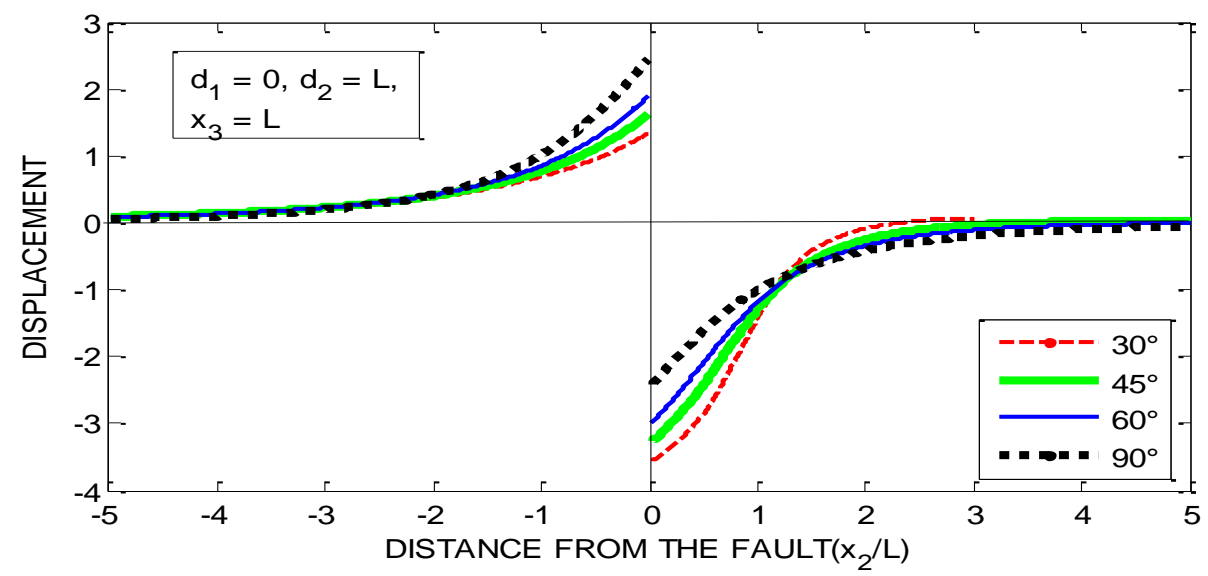

Figure 2. Variation of displacement $\left(u_{1} / b\right)$ with distance from the fault $\left(x_{2} / L\right)$. 


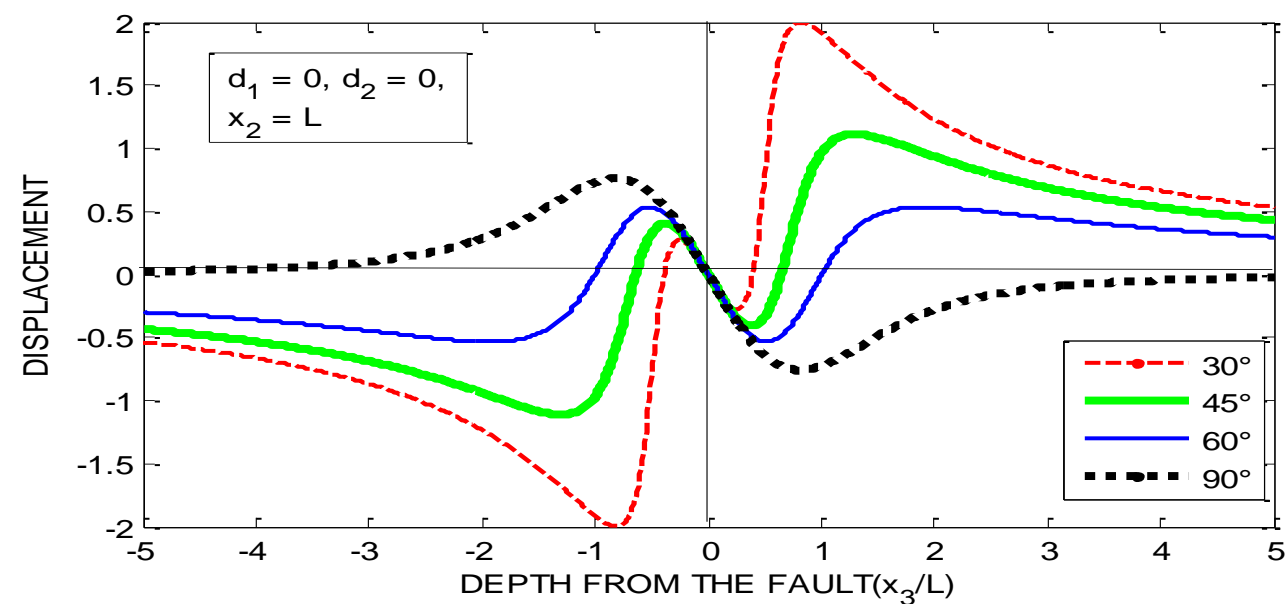

$3(\mathrm{a})$

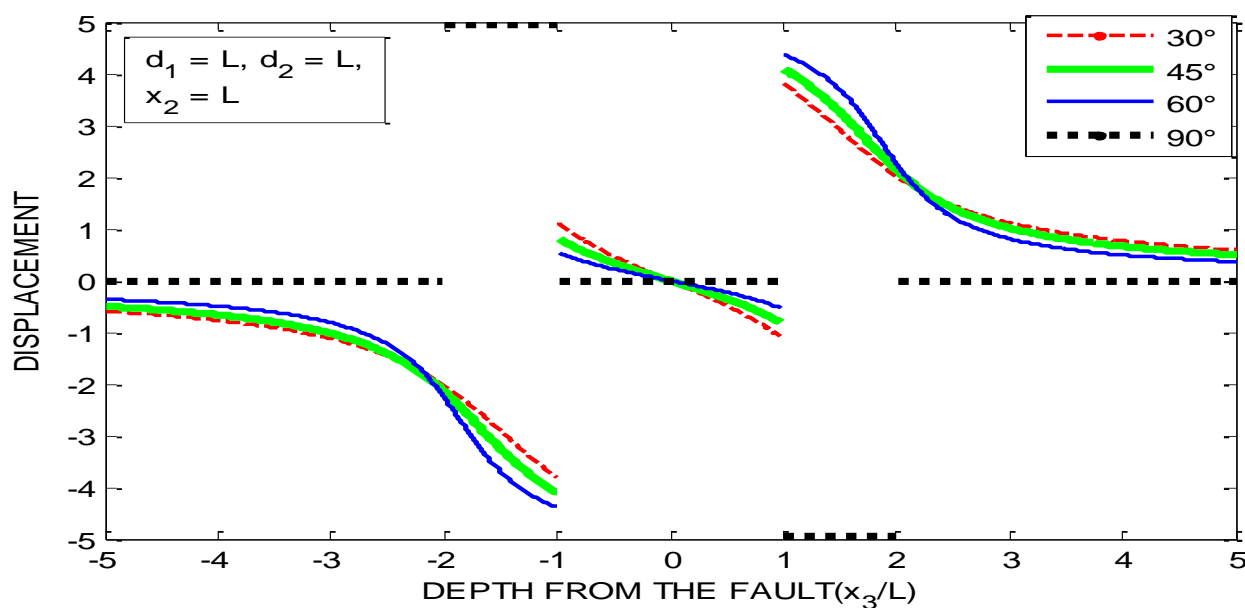

3(b)

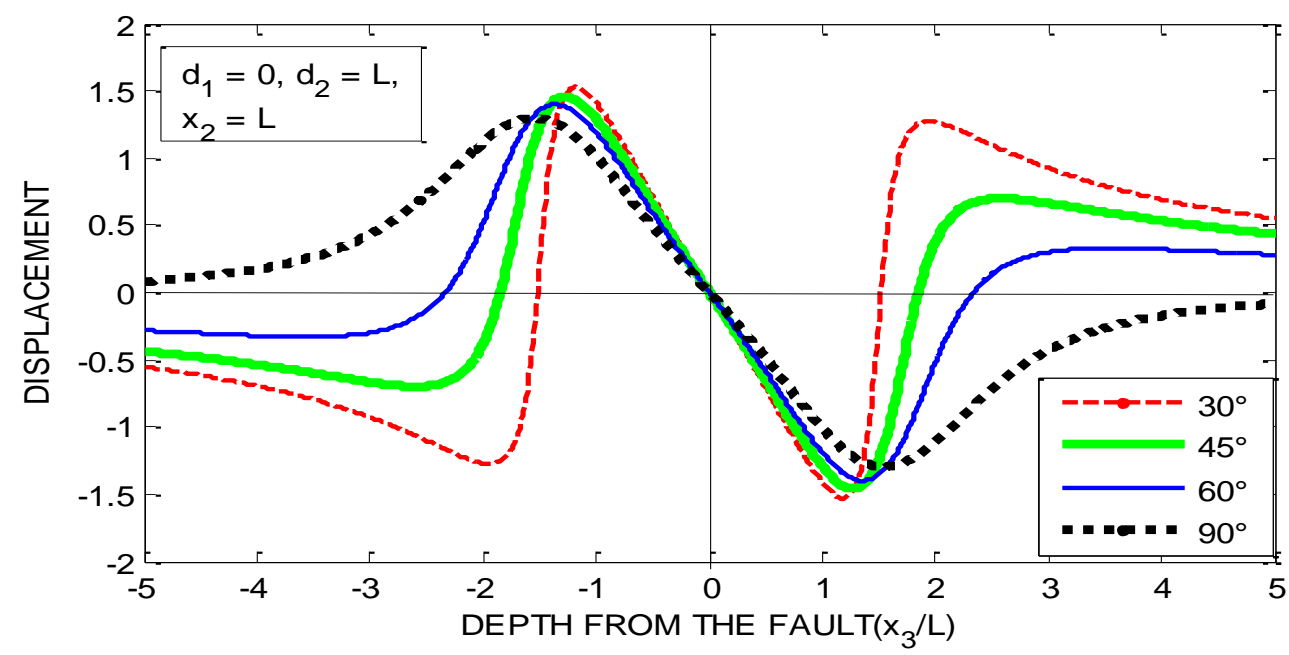

Figure 3. Variation of displacement $\left(u_{1} / b\right)$ with depth from the fault $\left(x_{3} / L\right)$. 


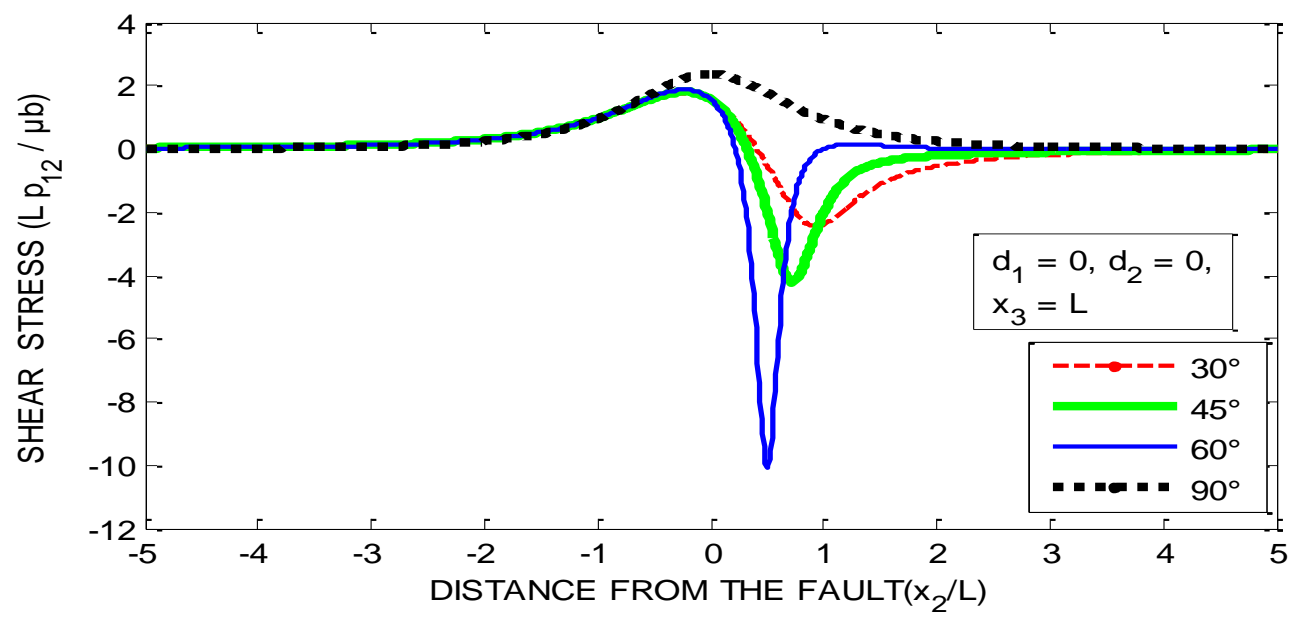

$4(\mathrm{a})$

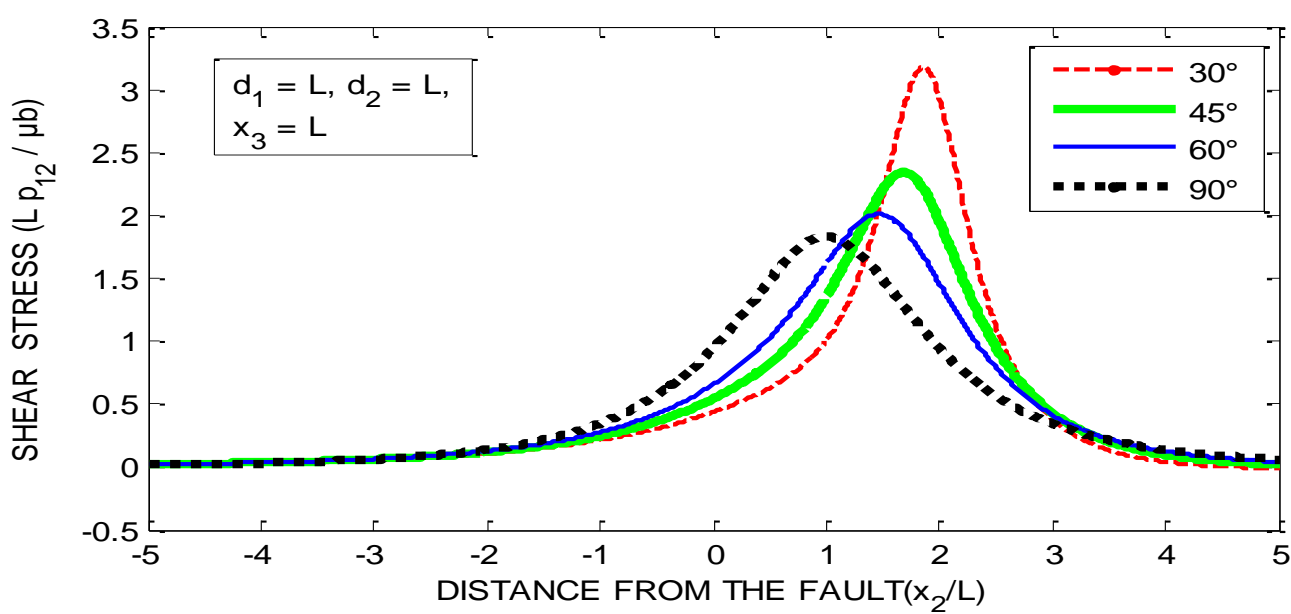

4(b)

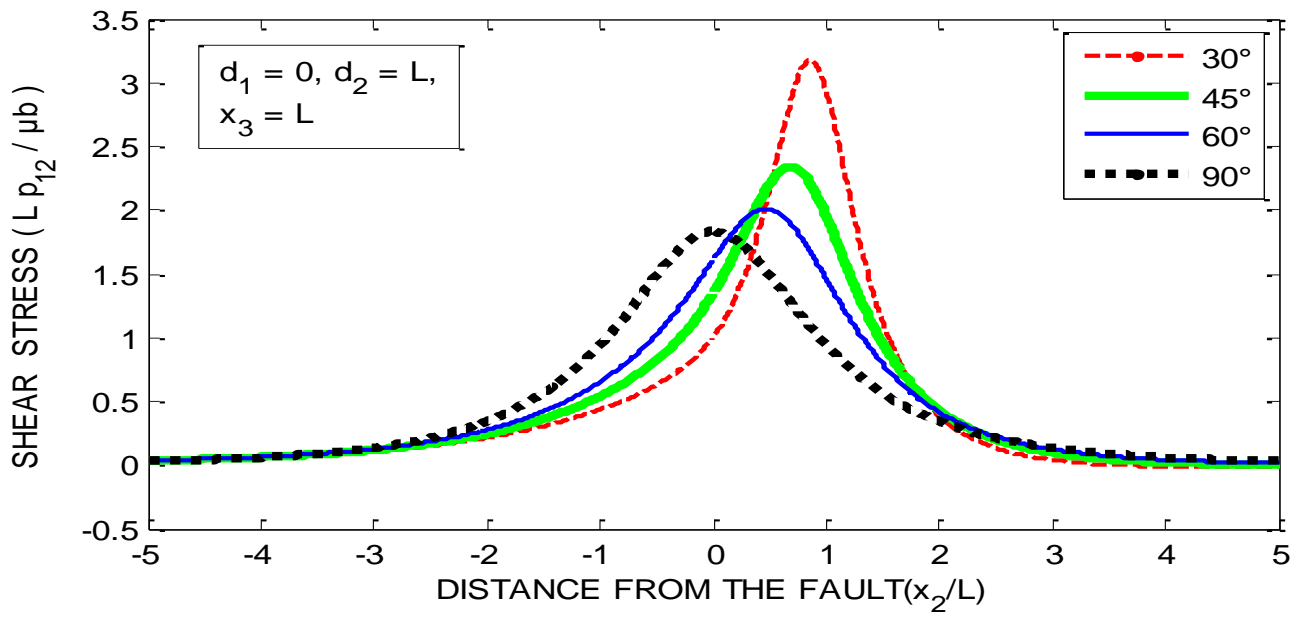

Figure 4.Variation of shear stress $\left(L p_{12} / \mu b\right)$ with distance from the fault $\left(x_{2} / L\right)$. 


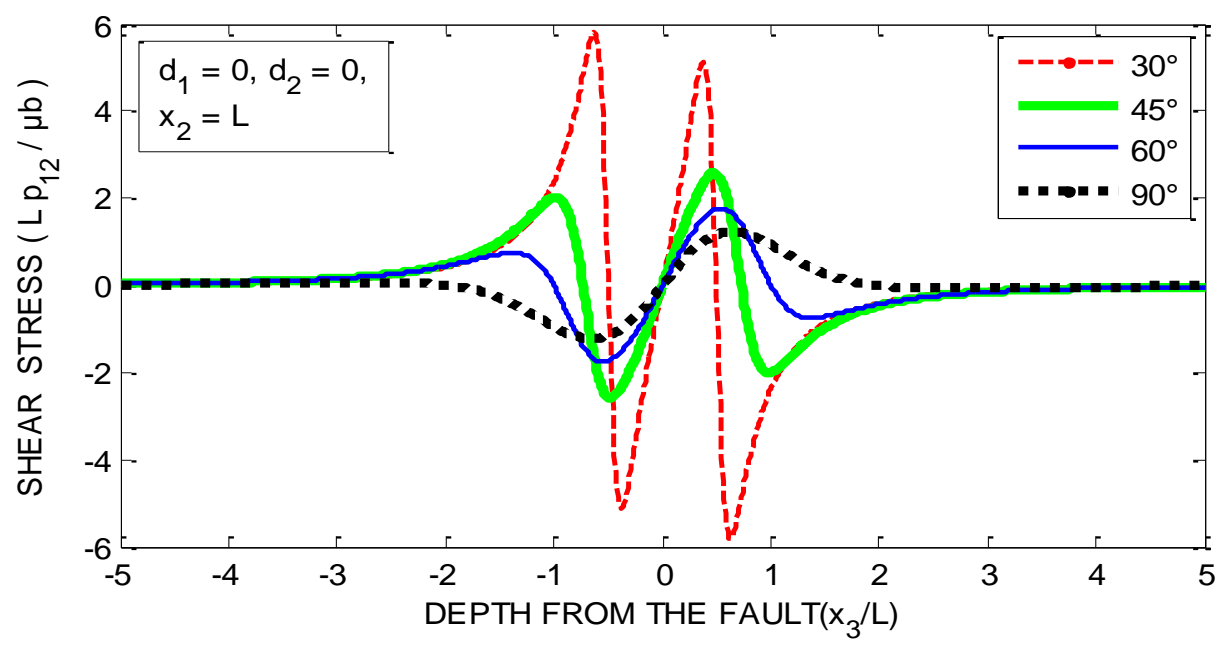

$5(a)$

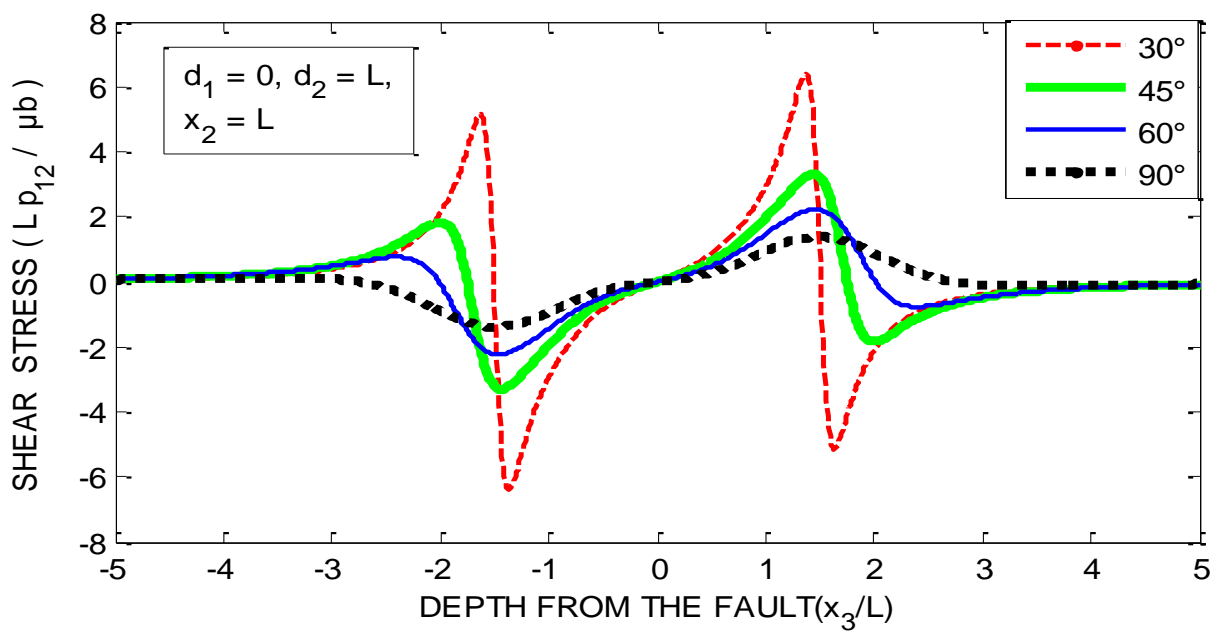

5 (b)

Figure 5.Variation of shear stress $\left(L p_{12} / \mu b\right)$ with depth from the fault $\left(x_{3} / L\right)$ for different values of dip angle $\delta=30^{\circ}, 45^{\circ}, 60^{\circ}, 90^{\circ}$ for $x_{2}=L$

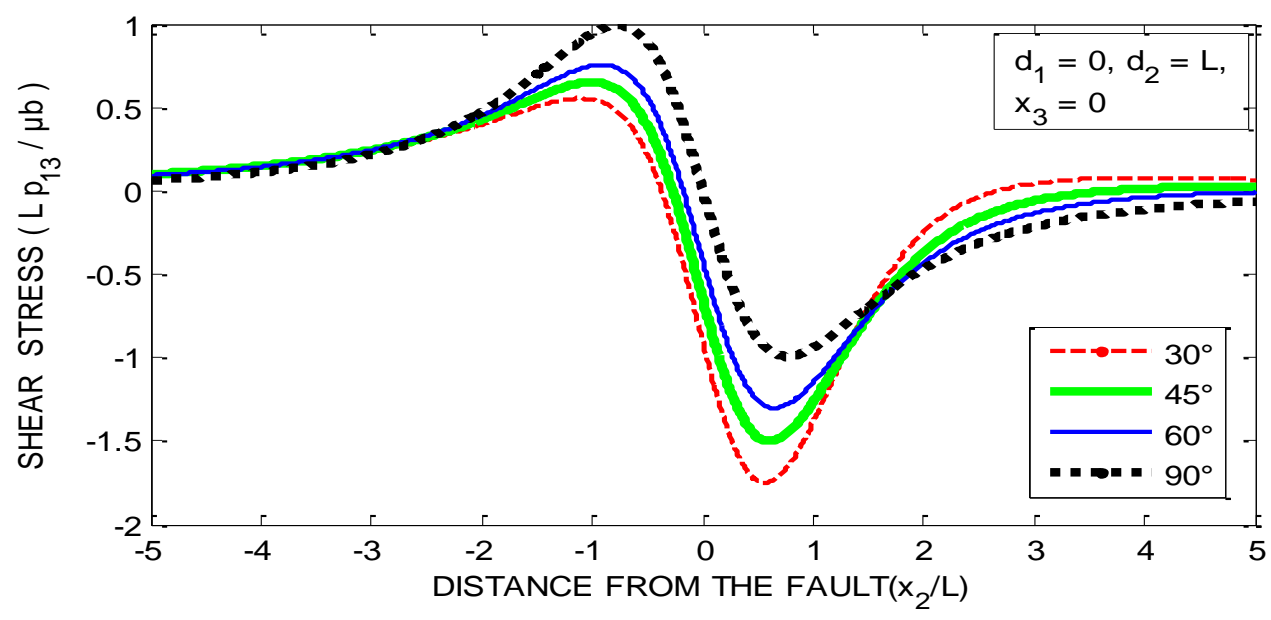




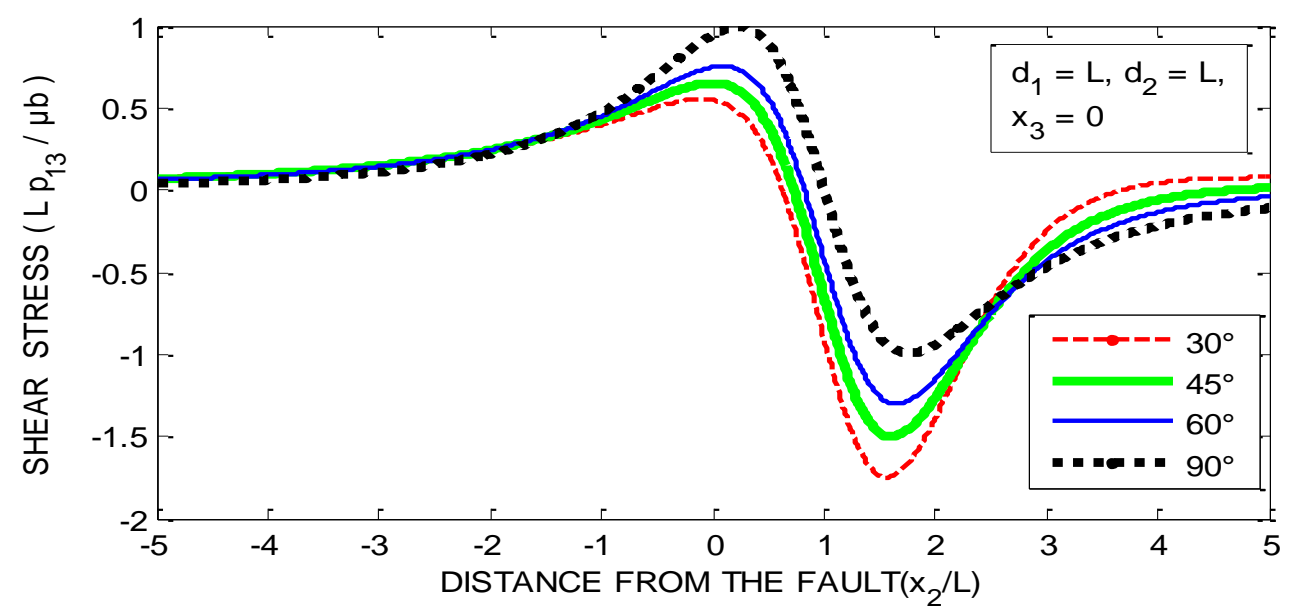

Figure 6.Variation of shear stress $\left(L p_{13} / \mu b\right)$ with distance from the fault $\left(x_{2} / L\right)$

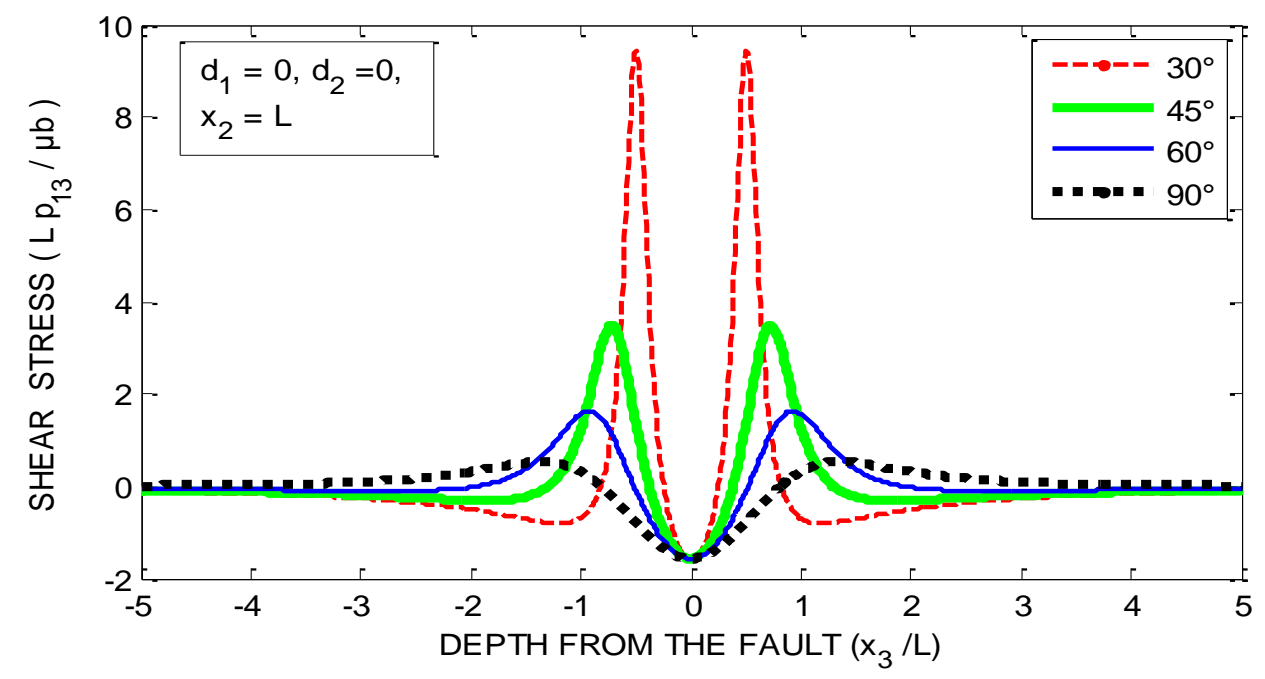

7(a)

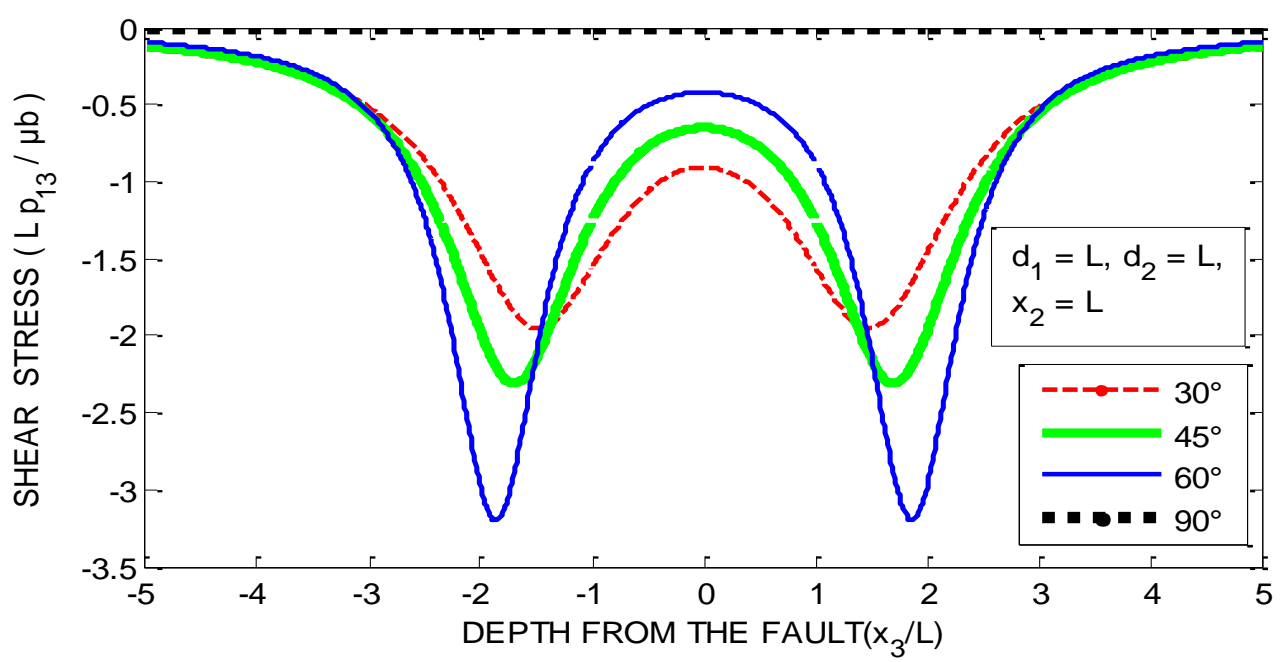

7(b) 


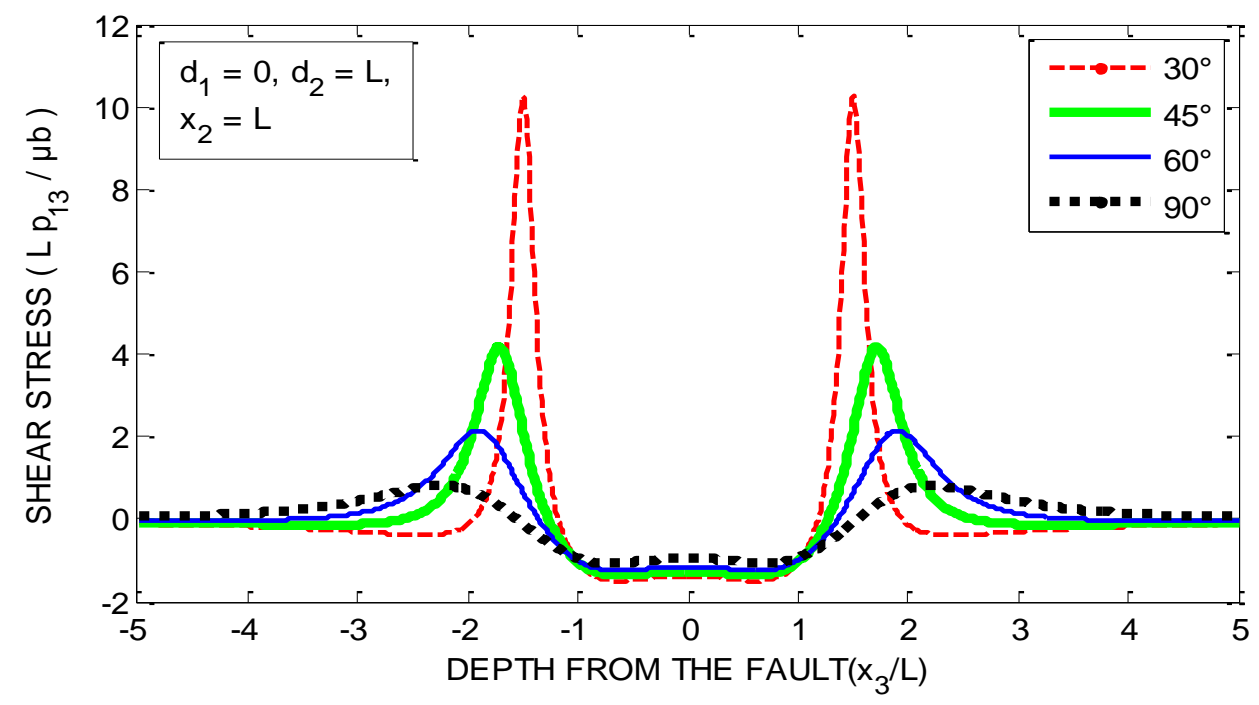

Figure 7.Variation of shear stress $\left(L p_{13} / \mu b\right)$ with depth from the fault $\left(x_{3} / L\right)$.

\section{Conclusion}

Analytical expressions for elastic residual field caused by a long inclined strike-slip fault of finite width $L$ located in a uniform half-space with rigid boundary have been obtained. If we take the particular case of Rani et al. (1993) such as when one of the isotropic half-spce is removed, i.e., if we take, $\mu_{2}=0$, then $\mathrm{m} \rightarrow \infty$ and we will get a uniform half-space with rigid boundary. Our results have been verified with displacement and stress fields obtained by Rani et al (1993), for a particular case. The closed-form expressions are used to study the variation of the displacement and stresses at the interface with the distance from the fault and depth from the fault. The inclination of the fault introduces asymmetry of various degrees in the displacement field depending on the dip-angle. This model is useful when the medium on the other side of the material discontinuity is very hard. High-rigidity layers are generally present at depth below a volcanic edifice, covered by much softer volcanic-sedimentary layers composed of a mixture of ash, mud and lava (Bonafede \& Revalta, 1999). Results obtained may find applications in geophysical phenomenon for above mentioned areas.

\section{Acknowledgement}

I, Yogita Godara (JRF), am grateful to the council of scientific and Industrial Research, New Delhi for financial support. The authors are thankful to the referees for their comments which led to an improvement in the presentation of the paper .

\section{References}

[1]. Steketee, J.A, On Volterra's Dislocations in a Semi-infinite Elastic Medium, Can. J. Phys., vol. 36,1958a, 192-205.

[2]. Stekettee, J.A, Some Geophysical Applications of the Elasticity Theory of Dislocations, Can. J. Phys., vol. 36,1958b, 1168-1198.

[3]. Chinnery, M. A., The Deformation of the Ground Around Surface Faults, Bull. Seismol. Soc. Am., vol. 61, 1961, 355-372.

[4]. Maruyama, T., On Two-dimensional Elastic Dislocations in an Infinite and Semi-infinite Medium, Bull. Earthq. Res. Inst., vol. 44, $1966,811-871$

[5]. Wang, R. and Wu, H. L., Displacement and Stress Fields Due to a Non-uniform Slip Along a Strike-Fault, Pure Appl. Geophys., vol. 121, 1983, 601-609.

[6]. Singh, S. J., Static Deformation of a Multilayered Half-space by Two-dimensional sources, Acta Geophys. Pol., vol. 33, 1985, 123134.

[7]. Singh, S.J. and Garg, N.R, On the Representation of Two-Dimensional Seismic Sources, Acta Geophys. Pol., vol. 34, 1986, 1-12.

[8]. Sharma, R. K. and Garg, N. R., Subsurface Deformation of a Layered Half-space Due to a very Long Strike-Slip Dislocations, Proc. Ind. National Sci. Acad, 1991.

[9]. Rani, S. and Singh, S., Residual Elastic field in two welded half-spaces caused by along inclined Strike-slip Fault, Proc. Indian Acad. Sci.(Earth Planet. Sci.), vol. 59.A, , 1993, 455-464.

[10]. Madan, D.K. and Garg, N.R., Static Deformation of an Orthotropic Horizontal Elastic Layer Coupling in Different ways to a base due to a very Long Inclined Strike-slip Fault Embedded in a Layer, Indian J. pure. Appl. Math., vol. 28, 1997, 697-712.

[11]. Bonafede, M.and Rivalta, E. (1999). The Tensile Dislocation Problem in a Layered Elastic Medium, Geophysical Journal International, Vol. 136, pp. 341-356.

[12]. Kumar, A., Singh, S. and Singh, J., Static Deformation of Two Welded Monoclinic Elastic Half-spaces due to a Long Inclined Strike-slipFault, Proc.of Indian Acad. Of Sci-Earth and Planet. Sci., vol. 111, 2002, 125-131.

[13]. Chugh, S., Madan, D. K. and Singh, K., Static Deformation Due To Non-Uniform Slip Along a Very Long Vertical Strike-Slip Geological Fault In Two- Phase Medium, Int. J. of Ecological Economics and Statistics, vol. 19, 2010, 47-65.

[14]. Singh, J., Malik, M. and Singh, M., Deformation of a uniform half-space with rigid boundary due to strike-slip line source, IOSR-J. Math., vol. 4, 2013, 26-37. 